\title{
ASTROPHYSICAL APPLICATIONS OF THE THEORY OF SCALE RELATIVITY
}

\author{
LAURENT Nottale ${ }^{a}$ \\ a CNRS, LUTH, Observatoire de Paris-Meudon, F-92195 Meudon Cedex, France
}

\begin{abstract}
In the framework of the theory of scale relativity, one considers the possibility that space-time be not only curved but also fractal at large scales. Then one can show that the equation of dynamics in such a space (i.e. the geodesics equation), takes the form of a generalized Schrödinger equation. This approach allows one to suggest new solutions to the problems of the formation and evolution of gravitational structures and of the 'missing mass'. Indeed, such a description leads to a natural self-organization process and involves the apparition of a new potential energy which is a manifestation of the fractal geometry (in similarity with the Newtonian potential being a manifestation of curvature). We suggest that this 'dark potential' is the cause of the various effects that have up to now been attributed to a postulated non-baryonic dark matter. We conclude by a brief survey of various observational effects that come in support of such a proposal.
\end{abstract}

\section{Introduction}

In its present acceptance, gravitation is understood as the various manifestations of the geometry of space-time at large scales. Up to now, in the framework of Einstein's theory [1], this geometry was considered to be Riemannian, i.e. curved. However, in the new framework of scale relativity, the geometry of space-time is assumed to be characterized not only by curvature, but also by fractality [2] beyond a new relative time-scale and/or space-scale of transition, which is an horizon of predictibility for the classical deterministic description. While the concept of fractal space-time has been first introduced in connection with the microscopic quantum theory $[3,4,5,6,7]$, it can indeed be also applied to the macroscopic realm, but with a different interpretation. Now, fractality manifests itself, in the simplest case, in terms of the appearance of a new scalar field. We have suggested that this new field leads to spontaneous self-organization and may also be able to explain $[11,18]$, without additional non-barionic matter, the various astrophysical effects which have been, up to now, attributed to unseen "dark" matter . 


\section{Gravitational Schrödinger equation}

Let us first briefly recall the basics of the scale-relativistic theoretical approach. Under three general conditions, namely, $\{(\mathrm{i})$ infinity of geodesics (which leads to introduce a non-deterministic velocity field), (ii) fractal dimension $D_{F}=2$ of each geodesic, on which the elementary displacements are described in terms of the sum $d X=d x+d \xi$ of a classical, differentiable part $d x$ and of a fractal, non-differentiable fluctuation $d \xi$, (iii) two-valuedness of the velocity field, which is a consequence of time irreversibility at the infinitesimal level issued from non-differentiability , one can construct a complex covariant derivative that reads

$$
\frac{\bar{d}}{d t}=\frac{\partial}{\partial t}+\mathcal{V} \cdot \nabla-i \mathcal{D} \Delta
$$

where $\mathcal{D}$ is a parameter that characterizes the fractal fluctuation, which is such that $\left\langle d \xi^{2}\right\rangle=2 \mathcal{D} d t$, and where the classical part of the velocity field, $\mathcal{V}$ is complex as a consequence of condition (iii) (see [8] for a recent complete demonstration).

Then this covariant derivative, that describes the non-differentiable and fractal geometry of space-time, can be combined with the covariant derivative of general relativity, that describes the curved geometry. We shall briefly consider in what follows only the Newtonian limit. In this case the equation of geodesics keeps the form of Newton's fundamental equation of dynamics in a gravitational field,

$$
\frac{\bar{D} \mathcal{V}}{d t}=\frac{\bar{d} \mathcal{V}}{d t}+\nabla\left(\frac{\phi}{m}\right)=0,
$$

where $\phi$ is the Newtonian potential energy. Introducing the action $S$, which is now complex, and making the change of variable $\psi=e^{i S / 2 m \mathcal{D}}$, this equation can be integrated under the form of a generalized Schrödinger equation [3]:

$$
\mathcal{D}^{2} \Delta \psi+i \mathcal{D} \frac{\partial}{\partial t} \psi-\frac{\phi}{2 m} \psi=0
$$

Since the imaginary part of this equation is the equation of continuity (Sec. 3), and basing ourselves on our description of the motion in terms of an infinite family of geodesics, $P=\psi \psi^{\dagger}$ naturally gives the probability density of the particle position [8]. This result is supported by Hermann's numerical simulations [25].

Even though it takes this Schrödinger-like form, equation (3) is still in essence an equation of gravitation, so that it must come under the equivalence principle $[13,19]$, i.e., it is independent of the mass of the test-particle. In the Kepler central potential case $(\phi=-G M m / r), G M$ provides the natural length-unit of the system under consideration. As a consequence, the parameter $\mathcal{D}$ reads:

$$
\mathcal{D}=\frac{G M}{2 w},
$$

where $w$ is a constant that has the dimension of a velocity. The ratio $\alpha_{g}=w / c$ actually plays the role of a macroscopic gravitational coupling constant $[19,17]$. 


\section{Formation and evolution of structures}

Let us now compare our approach with the standard theory of gravitational structure formation and evolution. By separating the real and imaginary parts of the Schrödinger equation we obtain, after a new change of variables, respectively a generalized Euler-Newton equation and a continuity equation, namely,

$$
m\left(\frac{\partial}{\partial t}+V \cdot \nabla\right) V=-\nabla(\phi+Q), \quad \frac{\partial P}{\partial t}+\operatorname{div}(P V)=0,
$$

where $V$ is the real part of the complex velocity field $\mathcal{V}$. In the case when the density of probability is proportional to the density of matter, $P \propto \rho$, this system of equations is equivalent to the classical one used in the standard approach of gravitational structure formation, except for the appearance of an extra potential energy term $Q$ that writes:

$$
Q=-2 m \mathcal{D}^{2} \frac{\Delta \sqrt{P}}{\sqrt{P}} .
$$

The existence of this potential energy, (which amount to the Bohm potential in standard quantum mechanics) is, in our approach, readily demonstrated and understood: namely, it is the very manifestation of the fractality of space, in similarity with Newton's potential being a manifestation of curvature.

In the case when actual particles achieve the probability density distribution (structure formation), we have $\rho=m_{0} P$; then the Poisson equation (i.e., the field equation) becomes $\Delta \phi=4 \pi G m m_{0} \psi \psi^{\dagger}$ and it is therefore strongly interconnected with the Schrödinger equation (which is here a new form for the equation of motion). Such a system of equations is similar to that encountered in the description of superconductivity (Hartree equation). We expect its solutions to provide us with general theoretical predictions for the structures (in position and velocity space) of self-gravitating systems at multiple scales $[10,18]$. This expectation is already supported by the observed agreement of several of these solutions with astrophysical observational data $[3,13,17,14,15,20,21,16]$.

\section{Examples of applications to astrophysics}

The theory has been able to predict in a quantitative way several new effects in the domain of gravitational structuring [18]. Moreover, these predictions have been successfully checked in various systems on a large range of scales and in terms of a common gravitational coupling constant (or one of its multiples or submultiples) whose value averaged on these systems was found to be $w_{0}=c \alpha_{g}=144.7 \pm 0.7$ $\mathrm{km} / \mathrm{s}[13]$. Indeed, new structures have been theoretically predicted, then checked by the observational data in a statistically significant way, for our solar system, including distances of planets $[3,14]$ and satellites $[16]$, sungrazer comet perihelions [18], obliquities and inclinations of planets and satellites [20], exoplanets semimajor axes [13, 17] (see Fig. 2) and eccentricities [18] (see Fig. 3), including planets 


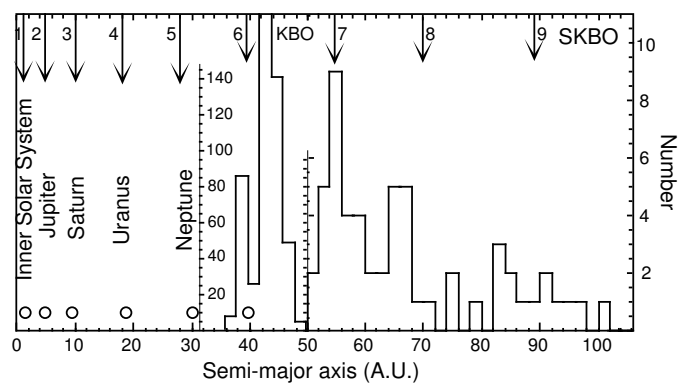

Figure 1: Distribution of the semi-major axis of Kuiper belt objects (KBO) and scattered Kuiper belt objects (SKBO), compared with the theoretical predictions (arrows) of probability density peaks for the outer solar system [18] (see text). The existence of probability density peaks for the Kuiper belt at $\approx 40,55,70,90 \mathrm{AU}$, etc..., has been theoretically predicted before the discovery of these objects [22], and it is now supported by the observational data.

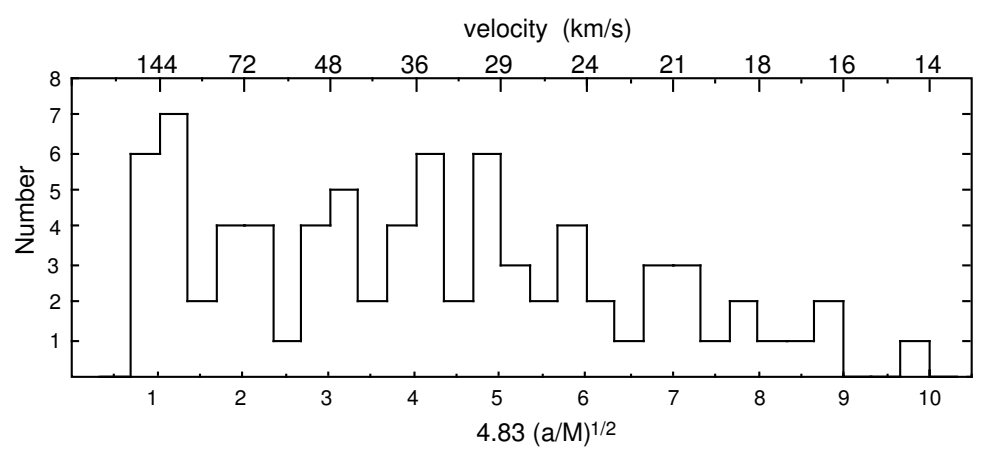

Figure 2: Observed distribution of the semi-major axes of recently discovered exoplanets and inner solar system planets, compared with the theoretical prediction. One predicts the occurence of peaks of probability density for semimajor axes $a_{n}=G M\left(n / w_{0}\right)^{2}$, where $n$ is integer, $M$ is the star mass and $w_{0}=144.7 \pm 0.7 \mathrm{~km} / \mathrm{s}$ is a gravitational coupling constant (see text). The probability to obtain such an agreement by chance is $P=4 \times 10^{-5}$.

around pulsars, for which a high precision is reached [13, 21], double stars [15], planetary nebula [18], binary galaxies [9] (see Fig. 4), our local group of galaxies [18], clusters of galaxies and large scale structures of the universe [15, 18].

Let us briefly consider the application of the theory to the formation of planetary systems. The standard model of formation of planetary systems can be reconsidered in terms of a fractal description of the motion of planetesimals in the protoplanetary nebula. On length-scales much larger than their mean free path, we have assumed [3] that their highly chaotic motion satisfy the three conditions upon which the derivation of a Schrödinger equation is based (large number of trajectories, fractality and time symmetry breaking). 


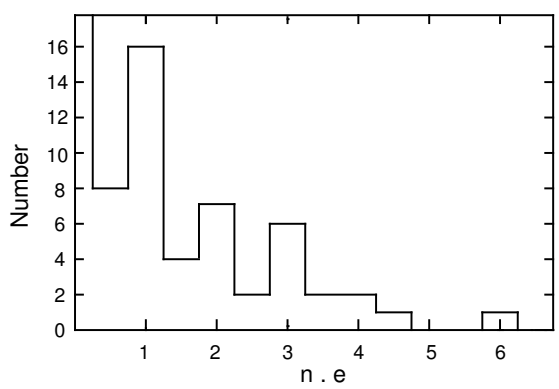

Figure 3: Observed distribution of the eccentricities of exoplanets. The theory predicts that the product of the eccentricity $e$ by the quantity $\tilde{n}=4.83(a / M)^{1 / 2}$, where $a$ is the semi-major axis and $M$ the parent star mass, should cluster around integers. The data support this theoretical prediction at a probability level $P=10^{-4}[18]$.

This description applies to the distribution of planetesimals in the protoplanetary nebula at several embedded levels of hierarchy. Each hierarchical level $(k)$ is characterized by a length-scale defining the parameter $\mathcal{D}_{k}$ (and therefore the velocity $w_{k}$ ) that appears in the generalized Schrödinger equation describing this sub-system. This hierarchical model has allowed us to recover the mass distribution of planets and small planets in the inner and outer solar systems [14]. It is generally supported by the structure of our own solar system, which is made of several subsystems embedded one in another, namely:

*The Sun. Through Kepler's third law, the velocity $w=3 \times 144.7=434.1$ $\mathrm{km} / \mathrm{s}$ is very closely the Keplerian velocity at the Sun radius $\left(R_{\odot}=0.00465 \mathrm{AU}\right.$ corresponds to $w_{\odot}=437.1 \mathrm{~km} / \mathrm{s}$ ). Moreover, one can also apply our approach to the organization of the sun surface itself. One expect the distribution of the various relevant physical quantities that characterize the solar activity at the Sun surface (sun spot number, magnetic field, etc...) to be described by a wave function whose stationary solutions read $\psi=\psi_{0} e^{i E t / 2 m \mathcal{D}}$.

The energy $E$ results from the rotational velocity and, to be complete, should also include the turbulent velocity, so that $E=\left(v_{\text {rot }}^{2}+v_{\text {turb }}^{2}\right) / 2$. This means that we expect the solar surface activity to be subjected to a fundamental period:

$$
\tau=\frac{2 \pi m \mathcal{D}}{E}=\frac{4 \pi \mathcal{D}}{v_{\text {rot }}^{2}+v_{\text {turb }}^{2}},
$$

The parameter $\mathcal{D}$ at the Sun radius is $\mathcal{D}=G M_{\odot} / 2 w_{\odot}$, then we obtain:

$$
\tau=\frac{2 \pi G M_{\odot}}{w_{\odot}\left(v_{\text {rot }}^{2}+v_{\text {turb }}^{2}\right)} .
$$

The average sideral rotation period of the Sun is 25.38 days, yielding a velocity of $2.01 \mathrm{~km} / \mathrm{s}$ at equator [23]. The turbulent velocity has been found to be $v_{t u r b}=$ $1.4 \pm 0.2 \mathrm{~km} / \mathrm{s}$ [24]. Therefore we find numerically

$$
\tau=(10.2 \pm 1.0) \text { yrs. }
$$


The observed value of the period of the Solar activity cycle, $\tau_{o b s}=11.0 \mathrm{yrs}$, supports this theoretical prediction. We shall in future works test this proposal by a more detailed study of the activity of the Sun and of other stars.

*The intramercurial system, organized on the constant $w_{\odot}=3 \times 144=432$ $\mathrm{km} / \mathrm{s}$. The existence of an intramercurial subsystem is supported by various stable and transient structures observed in dust, asteroid and comet distributions (see [18]). We have in particular suggested the existence of a new ring of asteroids, the 'Vulcanoid belt', at a preferential distance of about 0.17 AU from the Sun.

*The inner solar system (earth-like planets), organized with a constant $w_{i}=144 \mathrm{~km} / \mathrm{s}$ (see Fig. 2).

*The outer solar system (Jovian planets), organized with a constant $w_{o}=$ $144 / 5=29 \mathrm{~km} / \mathrm{s}$ (see Fig. 1), as deduced from the fact that the mass peak of the inner solar system lies at the Earth distance $(n=5)$. The recently discovered Kuiper and scattered Kuiper belt objects (Fig. 1) show peaks of probability at $n=6$ to 9 [18], as predicted before their discovery [22].

We have suggested more than ten years ago [3, 22], before the discovery of exoplanets, that the theoretical predictions from this approach should apply to all planetary systems, not only our own solar system. Meanwhile more than 120 exoplanets have been discovered, and the observational data support this prediction in a highly statistically significant way (see $[13,17,18]$ and Figs. 2 and 3).

A full account of this new domain would be too long to be included in the present contribution. We have given here only few typical examples of these effects (see the figures) and we refer the interested reader to the review paper Ref. [18] and references therein for more detail.

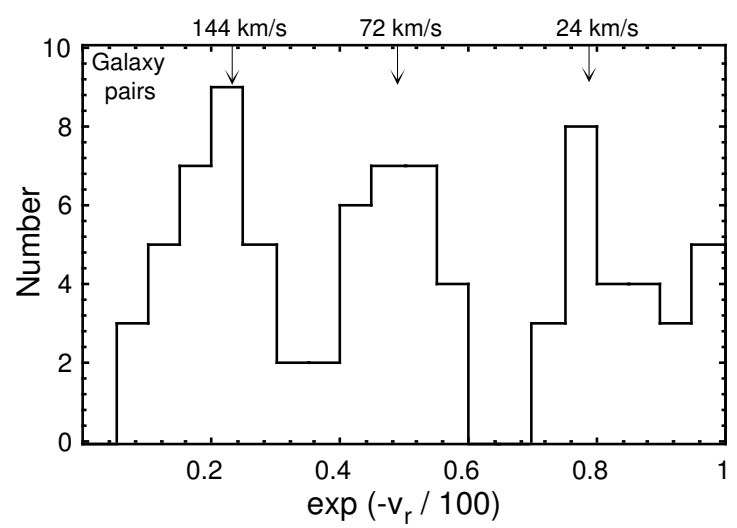

Figure 4: Deprojection of the intervelocity distribution of galaxy pairs (Tricottet and Nottale, reported in [18]) from the Schneider-Salpeter catalog with precision redshifts. The main probability peak is found to lie at $144 \mathrm{~km} / \mathrm{s}$ (plus secondary peaks at $72=144 / 2 \mathrm{~km} / \mathrm{s}$ and $24=144 / 6$ $\mathrm{km} / \mathrm{s}$ ), in agreement with the exoplanet and inner solar system structuring (see Fig. 2). 


\section{Possible solution to the "dark matter" problem}

In the case (ii) of isolated test particles, the density of matter $\rho$ may be nearly zero while the probability density $P$ does exist, but only as a virtual quantity that determines the potential $Q$. In this situation, even though there is no or few matter at the point considered (except the test particles that are assumed to have a very low contribution), the effects of the potential $Q$ are real (since it is the result of the structure of the geodesics two-fluid).

We have therefore suggested $[11,18,12]$ that this extra scalar field, which is a manifestation of the fractality of space, may be responsible for the various dynamical and lensing effects which are usually attributed to unseen "dark matter". This interpretation is supported by the fact that, for a stationary solution of the gravitational Schrödinger equation, one gets the general energy relation $E=\phi+Q+\frac{1}{2} m V^{2}$, where $E / m$ can take only quantized values.

This result can be applied, as an example, to the motion of bodies in the outer regions of spiral galaxies. In these regions there is practically no longer any visible matter, so that the Newtonian potential (in the absence of additional dark matter) is Keplerian. While the standard Newtonian theory predicts for the velocity of the halo bodies $v \propto \phi^{1 / 2}$, i.e. $v \propto r^{-1 / 2}$, we predict $v \propto|(\phi+Q) / m|^{1 / 2}$, i.e., $v=$ constant. More specifically, assuming that the gravitational Schrödinger equation is solved for the halo objects in terms of the fundamental level wave function, one finds $Q_{\text {pred }}=-\left(G M m / 2 r_{B}\right)\left(1-2 r_{B} / r\right)$, where $r_{B}=G M / w_{0}^{2}$. This is the result systematically observed in spiral galaxies (i.e., flat rotation curves) which has motivated (among other effects) the assumption of the existence of dark matter.

\section{Conclusion}

We have recalled in the present contribution how the theory of scale relativity is able to yield equations that describe a natural tendency to make structures, as a very consequence of the fractality of space.

Moreover, we have suggested that the effects tentatively attributed to unseen matter are simply the result of this geometry of space-time. In this proposal, space-time is not only curved but also fractal beyond some given relative time and space-scales. While the curvature manifests itself in terms of the Newton potential, fractality would manifest itself in terms of the new scalar potential $Q$, and then finally in terms of the anomalous dynamics and lensing effects.

\section{References}

[1] A. Einstein, Annalen der Physik 49, 769 (1916), translated in The Principle of Relativity (Dover, 1923, 1952)

[2] B. Mandelbrot, The Fractal Geometry of Nature (Freeman, San Francisco, 1982) 
[3] L. Nottale, Fractal Space-Time and Microphysics: Towards a Theory of Scale Relativity (World Scientific, Singapore, 1993)

[4] L. Nottale and J. Schneider, J. Math. Phys. 25, 1296 (1984)

[5] G. N. Ord, J. Phys. A: Math. Gen. 16, 1869 (1983)

[6] L. Nottale, Int. J. Mod. Phys. A 4, 5047 (1989)

[7] M. S. El Naschie, Chaos, Solitons \& Fractals 2, 211 (1992)

[8] M.N. Célérier \& L. Nottale, J. Phys. A: Math. Gen. 37, 931(2004)

[9] L. Nottale, Chaos, Solitons \& Fractals 7, 877 (1996)

[10] L. Nottale, Astron. Astrophys. 327, 867 (1997)

[11] L. Nottale, in Frontiers of Fundamental Physics, Proceedings of Birla Science Center Fourth International Symposium, 11-13 december 2000, edited by B. G. Sidharth and M. V. Altaisky, (Kluwer Academic, 2001) p. 65

[12] L. Nottale, Chaos, Solitons and Fractals 16, 539 (2003)

[13] L. Nottale, Astron. Astrophys. Lett. 315, L9 (1996)

[14] L. Nottale, G. Schumacher and J. Gay, Astron. Astrophys. 322, 1018 (1997)

[15] L. Nottale and G. Schumacher, in Fractals and beyond: complexities in the sciences, edited by M. M. Novak (World Scientific, 1998) p. 149

[16] R. Hermann, G. Schumacher, and R. Guyard, Astron. Astrophys. 335, 281 (1998)

[17] L. Nottale, G. Schumacher, E.T. Lefèvre, Astron. Astrophys. 361, 379 (2000)

[18] D. Da Rocha, L. Nottale, Chaos, Solitons \& Fractals 16, 565 (2003)

[19] A.G. Agnese, R. Festa, Phys. Lett. A227, 165 (1997)

[20] L. Nottale, Chaos, Solitons \& Fractals 9, 1035 (1998)

[21] L. Nottale, Chaos, Solitons \& Fractals 9, 1043 (1998)

[22] L. Nottale, in "Chaos and Diffusion in Hamiltonian Systems", ed. D. Benest and C. Froeschlé, (Frontières, 1994), p. 173

[23] J.C. Pecker and E. Schatzman, Astrophysique générale (Masson, Paris, 1959)

[24] K.R. Lang, Astrophysical Formulae (Springer-Verlag 1980)

[25] R. Hermann, J. Phys. A: Math. Gen 30, 3967 (1997) 